\title{
Light dark matter investigation with CRESST
}

\author{
L. Pattavina for the CRESST Collaboration ${ }^{*}$ \\ Physik Department, Technische Universität München, Garching D-85748 - Germany \\ Gran Sasso Science Institute, L'Aquila I-67100 - Italy \\ E-mail: luca.pattavina@tum.de
}

\begin{abstract}
Cryogenic detectors are excellent devices for rare event searches. Thanks to their low energy thresholds, these detectors are at the cutting edge for the investigations of light dark matter (DM). The CRESST (Cryogenic Rare Event Search with Superconducting Thermometers) experiment explores with high sensitivity the parameter space of low mass DM candidates, being the pathfinder in the sub-GeV/c ${ }^{2}$ mass range. CRESST employs scintillating $\mathrm{CaWO}_{4}$ crystals as target material operated at $\mathrm{mK}$ temperature. Thanks to the simultaneous read-out of heat, in the main absorber, and light, with an auxiliary cryogenic light detector, the background in the region of interest is strongly suppressed. In 2018, the first stage of CRESST-III data taking was successfully completed, achieving an unprecedented energy threshold for nuclear recoils, lower than 100 eV. New results of CRESST-III will be presented accompanied by a status update on the ongoing activities.
\end{abstract}

Neutrino Oscillation Workshop (NOW2018)

9 - 16 September, 2018

Rosa Marina (Ostuni, Brindisi, Italy)

\footnotetext{
* Speaker.

${ }^{\dagger}$ Also at INFN - Laboratori Nazionali del Gran Sasso, Assergi (L’Aquila) I-67010 - Italy.
} 


\section{Introduction}

Last decades of astronomical and cosmological observations tell us that our knowledge of the Universe covers a minimum part of it: the Standard Model of particle physics describes only the $5 \%$ of the overall energy density components. The largest part, that accounts for the $68 \%$ of the total, seems to consist in the so-called Dark Energy, an unknown form of energy introduced to explain the accelerated expansion rate of the Universe. The remaining $27 \%$ appears to be made of non-baryonic and non-luminous matter, that for this reason is named Dark Matter (DM).

Besides the standard DM candidates, the Weakly Interacting Massive Particles [1], also lighter candidates were discussed during the last years. Examples are dark photons [2,3] with masses on the keV-scale or below, and asymmetric DM [4] with masses $\mathrm{m}_{D M}$ of the order of few $\mathrm{GeV} / \mathrm{c}^{2}$.

The strategy for their direct detection is based on the observation of the nuclear recoil produced by an elastic scattering between a DM candidate and a target nucleus. Given the small energy deposit and the low expected interaction rate, this search is performed with detectors featuring an ultra-low energy threshold and low-background level in the region of interest (RoI).

\section{The CRESST experiment}

Currently the cryogenic technology proved to be the best technique, among many (e.g. TPCs, scintillators, ...), for the study of light DM particles. Nowadays the CRESST experiment is the most sensitive having established the most stringent limit on the interaction cross section for sub-GeV/c ${ }^{2}$ DM particles.

CRESST is an acronym for Cryogenic Rare Event Search with Superconducting Thermometers. CRESST uses $\mathrm{CaWO}_{4}$ cryogenic calorimeters as target material for the investigation of nuclear recoils induced by DM interactions. The calorimeters are operated at a temperature of $10 \mathrm{mK}$ inside a dedicated cryogenic infrastructure. The $\mathrm{CaWO}_{4}$ crystals are equipped with Transition Edge Sensors (TES) for the read out of the positive temperature variations induced by particle interactions. $\mathrm{CaWO}_{4}$ is an efficient scintillator also at low temperatures, for this reason the main absorber are coupled to cryogenic light detectors (LD) to additionally measure the scintillation light. These devices are equipped with TES as well. The double read-out of heat and light allows for a particle identification, which is of paramount importance for the background suppression in the RoI.

The experiment is located in the Gran Sasso deep underground laboratory (L'Aquila, Italy) for mitigating the background induced by cosmic ray interactions. In fact about 3600 mw.e. of coverage ensures a muon flux suppression by a factor $10^{6}$ with respect to an above ground installation.

\section{TES-based cryogenic detectors}

The temperature sensors used in CRESST are Tungsten films operated as TES. TES ensure excellent sensitivity to low energy particle interactions, in fact they are superconducting films operated in between the normal-to-superconducting phase. In this regime a small temperature variation induces a large increase in the resistance of the film. Details on the operations of CRESST TES can be found in [5]. 
Each $\mathrm{CaWO}_{4}$ crystal is paired with a $\mathrm{LD}$ made of am $\mathrm{Al}_{2} \mathrm{O}_{3}$ wafer on which a $1 \mu \mathrm{m} \mathrm{SiO}_{2}$ layer is evaporated, so to increase the light collection efficiency.

In the latest phase of the experiment specific features were included in the detector design, aiming at increasing the sensitivity of CRESST detectors towards low mass DM regions. Two main approaches were adopted: 1) suppress the background in the RoI and 2) improve the detector energy threshold. In Fig. 1, a CRESST-III detector module is shown, of which a total of 10 were used for the $1^{\text {st }}$ phase of CRESST-III data taking.

To address the background suppression issue a

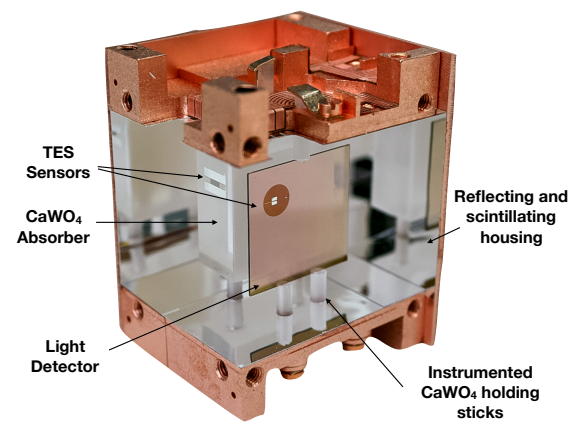

Figure 1: CRESST-III detector module. The various features of the detector are visible: fully scintillating housing, instrumented detector holders and TES sensors optimized for each absorber (phonon and light detectors). fully active detector housing was designed, so to identify possible radioactive decays occurring on the detector surfaces or housing. In fact a relevant background in the RoI is caused by radioactive contaminations (low energy $\beta$ and $\alpha$ decays) decaying on the detector surfaces [6]. Furthermore all the detector holding components, which are in direct contact with the detectors, were designed to be active. For this reason specific $\mathrm{CaWO}_{4}$ holders were designed, and instrumented with TES. These allowed for the identification of radioactive decays occurring on the holders and not in the bulk of the main detectors.

The second main improvement of CRESST-III detectors was the optimization of the energy threshold. In fact, while searching for direct light DM interactions, having low energy threshold is of paramount importance to probe the region of the parameter space where light DM candidate are allowed. This was the driving motivation for designing TES optimized for low energy threshold, and for having $24 \mathrm{~g}$ absorber crystals (about x10 smaller compared to CRESST-II [7]). Operating smaller size detectors with appropriate TES enables to reduce the overall heat capacity of the cryogenic system and thus to improve the detector energy threshold.

\section{Results of CRESST-III}

The first phase of CRESST-III started in July 2016 and continued until February 2018. The latest results on the low energy analysis focused mostly on data taking period between October 2016 and May 2017. Thanks to the excellent performance of the detector and to an optimum trigger algorithm [8] an unprecedented energy threshold was achieved, as shown in Fig. 2. The best performing detector had an energy threshold of $26 \mathrm{eV}$, never achieved before with a $\mathrm{CaWO}_{4}$ crystal (24 g mass).

The data release is relative to Det-A, the best performing one, and the total collected statistics is $5.7 \mathrm{~kg} \cdot \mathrm{d}$. A first level analysis was performed on a sub-set of the entire statistics (20\%), blinding the remaining. On this sub-set the event selection cuts were defined. Finally, these cuts were applied on the entire statistics, the results are shown in Fig. 3. The total background energy spectrum is shown in grey, while the events that survive a Light Yield cut, which are those found in the RoI, are highlighted in red. We clearly identify an excess of events near the detector energy threshold, 


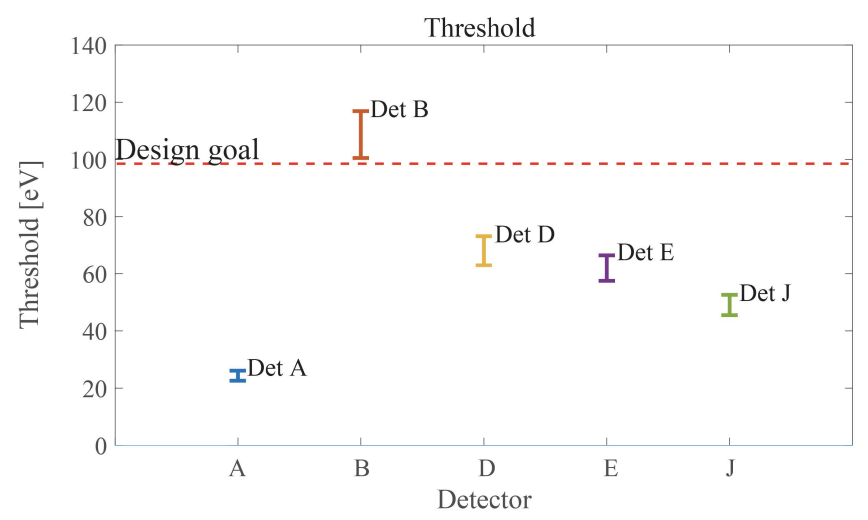

Figure 2: Energy threshold for the best performing CRESST-III detectors. The error bars are not uncertainty but refer to the noise-trigger rate acceptance, which here is set between 1 and 100 noise-triggered event over an exposure of $1 \mathrm{~kg} \cdot \mathrm{d}$ of exposure.

which after a preliminary analysis seems not to be compatible with a DM signal. In fact, if we compare this signal with the other detectors operated in the same configuration, this excess seems not to be compatible among different detectors. In Fig. 3, on the right the corresponding limit on the DM-nucleon interaction cross section is shown. This new CRESST-III limit improves the previous CRESST limit for all DM masses and extends the sensitivity to unprecedentedly low-energetic DM interactions, establishing the strongest limit for sub-GeV/c ${ }^{2} \mathrm{DM}$ candidates.

\section{References}

[1] L. Roszkowski et al., Rept. Prog. Phys. 81, 066201 (2018).

[2] M. Pospelov et al., Phys. Rev. D 78, 115012 (2008).

[3] H. An et al., Phys. Lett. B 747, 331 (2015)

[4] K. Petraki and R.R. Volkas, Int. J. Mod. Phys. A 28, 1330028 (2013).

[5] G. Angloher et al., (CRESST Coll.), 2015, Arxiv:1503.08065.

[6] M. Clemenza et al., Eur. Phys. J. C71 (2011) 1805.

[7] G. Angloher et al., (CRESST Coll.), Eur. Phys. J. C 74, 3184 (2014).

[8] C. Alduino et al., (CUORE Coll.), Eur. Phys. J. C 77, 857 (2017). 

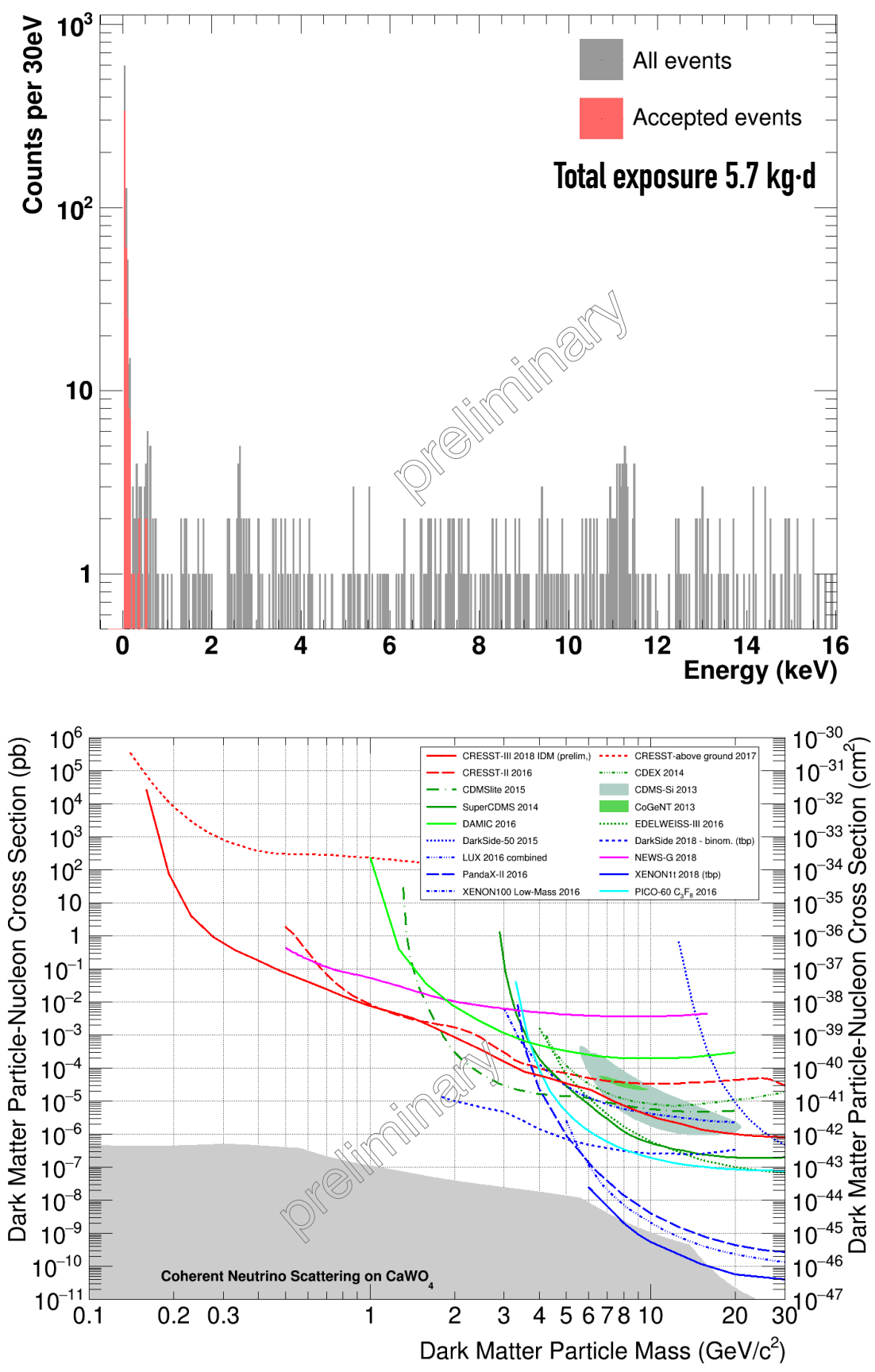

Figure 3: Results of the statistics acquired on Det-A after $5.7 \mathrm{~kg} \cdot \mathrm{d}$ of exposure. On the top the total energy spectrum is shown, and the events that populates the nuclear recoil RoI are highlighted in red. On the bottom the corresponding limit on the DM-nucleon interaction cross section is depicted. 